\title{
Diagnostic Value of HE4, CA125 and Risk of Ovarian Malignancy Algorithm in Detecting Ovarian Cancer
}

\author{
Mehri Jafari-Shobeiri ${ }^{1}$, Marzye Parizad ${ }^{1 *}$, Fatemeh Nazari ${ }^{1}$, Elaheh Ouladsahebmadarek ${ }^{1}$, Manizheh Sayyah-Melli ${ }^{1}$, \\ Parvin Mostafa-Gharabaghi ${ }^{1}$, Heidarali Esmaili ${ }^{2}$, Mohammad Amin Parizad ${ }^{3}$, Yasmin Pouraliakbar ${ }^{3}$, Farnaz Sepasi ${ }^{3}$
}

\begin{abstract}
Objectives: Ovarian cancer is one of the most common causes of cancer related death in women. Diagnostic techniques to detect ovarian cancer in the earlier stages are likely to improve prognosis. The aim of this study is to evaluate a novel serum tumor marker HE4 in comparison with CA125 and the risk of ovarian malignancy algorithm (ROMA) in patient with ovarian malignancy and benign tumors.

Materials and Methods: CA125, HE4, ROMA were determined in 33 patients with ovarian cancer and in 67 patients with benign tumors. CA125, HE4 cut offs were $35 \mathrm{U} / \mathrm{ml}$ and $70 \mathrm{pM}$, respectively. ROMA algorithm cut off was $13.1 \%$ and $27.7 \%$ for pre-menopausal or post-menopausal women, respectively. Data were analyzed by SPSS19 software; and $P<0.05$ was considered significant.

Results: In this study, serum level of CA125, HE4 and ROMA were higher in patients with ovarian cancer than benign tumors $(P<.001)$. Area under the ROC curve for CA125, HE4 and ROMA were $82.4 \%, 84.01 \%$ and $82.4 \%$, respectively. The differences were not significant.

Conclusion: The median CA125, HE4 and ROMA serum levels differed significantly between benign and malignant cases. Although the initial reports were promising, measurement of HE4 and ROMA did not increase the detection of malignant diseases compared with CA125 alone.

Keywords: CA125 antigen, HE4 protein, Ovarian neoplasms
\end{abstract}

\section{Introduction}

Surgery for benign ovarian mass in general hospital by a gynecologist seems to be adequate but those patients diagnosed with ovarian cancer have much better outcome if undergo surgery with oncologist-gynecologist (1). Therefore, it is of vital importance that risk of ovarian cancer in patients with pelvic mass be assessed so that patients who are likely high risks for cancer could be referred to appropriate centers. Currently, tumor markers CA125 is frequently used to detect ovarian cancer before clinical signs appear, but CA125 can increase in association with some physiological conditions such as in pre-menopausal women and in women having benign diseases (2). Much effort has been done in order to discover other markers supplementing CA 125 and or marker replacements. In 1999, Human epididimis protein 4 (HE4) gene expression in ovarian cancer has been cited (3). In several studies conducted for the purpose of determining the risk of cancer algorithm (risk of ovarian malignancy algorithm [ROMA]), combined levels of CA125 and HE4 in patients with ovarian cyst or pelvic mass were used to differentiate patients as low risk and high risk groups for ovarian neoplasm and in this manner, patients will be referred to centers that could offer the best care possible $(4,5)$. Considering that referral of patients to a well equipped gynecologic oncology center requires differentiation between benign and malignant ovarian neoplasm, therefore, in this study, the measurements of CA125 and HE4 markers and ROMA values were used in order to diagnose patients with neoplasm of the ovaries and to refer these patients to gynecologic oncology centers.

\section{Materials and Methods}

From the 21st of January to December 2014, 100 patients diagnosed with ovarian masses through sonography and scheduled for surgery were enrolled in this study. Those patients having history of any type of cancer and or having history of previous chemotherapy were excluded. 33 patients diagnosed with ovarian masses through sonography with suspicion of ovarian cancer through clinical and laboratory data were chosen for the malignant group; while 67 patients diagnosed with benign tumors based on the above-mentioned methods were selected for the benign group. Both these 2 groups were considered as the

Received 20 March 2015, Accepted 6 August 2015, Available online 1 October 2015

${ }^{1}$ Women's Reproductive Health Research Center, Tabriz University of Medical Science, Tabriz, Iran. ${ }^{2}$ Department of Pathology, Tabriz University of Medical Science, Tabriz, Iran. ${ }^{3}$ Tabriz University of Medical Science, Tabriz, Iran.

*Corresponding author: Marzye Parizad, Alzahra Hospital, Tabriz University of Medical Science, Tabriz, Iran. Tel: +9835541221, Email: parizad116@gmail.com 
study groups. Finally, the differentiations of ovarian masses from benign to malignant were based from the pathologist's report. Patients diagnosed with ovarian cancer were differentiated based on the Federation of Obstetrics and Gynecology Guidelines (FIGO) (6) then the type, stage and grade of tumor were determined.

After explaining the aim of the study to the subjects and after obtaining their written consent and ensuring about the confidentiality of their personal information, $5 \mathrm{ml}$. of blood was collected from both groups a day before surgery. Blood samples were immediately centrifuged at around $3000 \mathrm{rpm}$ for 10 minutes at $4^{\circ} \mathrm{C}$; the supernatant serum was collected and kept at $-70^{\circ} \mathrm{C}$ up to the time when the study population's HE4, CA125 and ROMA were tested. Sampling intervals and freezing were at a maximum of one hour. After determining the serum values of HE4 and CA125, the ROMA was calculated using the two tumor markers. Measurement of the series of CA125 and HE4 values were performed using chemiluminescent enzyme immunoassay and electro chemiluminescent enzyme immunoassay, respectively.

ROMA was calculated based on the following formula: Pre-menopausal predictive index $(\mathrm{PZ})=-12+2.38 \mathrm{LN}$ $(\mathrm{HE} 4)+0.0626 \times \mathrm{LN}(\mathrm{CA} 125)$ and post-menopausal predictive index $(\mathrm{PZ})=-8.09+1.04 \times \mathrm{LN}(\mathrm{HE} 4)+0.732 \times \mathrm{LN}$ (CA125). The cut off value of $35 \mathrm{u} / \mathrm{ml}$ for CA125 and HE4 based on the study of Nolen et al (7) and a pM of 70 was considered.

ROMA cuff off for patients with high risk of ovarian cancer in pre-menopausal women were considered as $\geq 13.1 \%$ and for menopausal women as $\geq 27.7 \%$, respectively based on the study conducted by Moore et al (4). Based on ROMA result subjects were differentiated into low risk and high risk groups. Data from this present study was gathered using the following methods; descriptive statistics (mean $\pm \mathrm{SD}$ ), Fisher exact test, chi-square test, ROC curve and calculation of sensitivity-specificity. Statistical analyses were performed using SPSS 19 with normal results. Distribution of data was evaluated with the use of Kolmogorov-Smirnov test. $P$ value of less than 0.05 was considered statistically significant.

\section{Results}

From the 67 patients with benign ovarian tumors, the most common histological type was endometrioma (29.9\%) (Table 1). Serous adenocarcinoma is the most common malignant tumor of the ovary (48.5\%), stage 3 (57.6\%) and grade $3(90.9 \%)$ were the most common stage and grade, respectively (Table 2). Average HE4 in patients with malignant ovarian tumors was $278.33 \pm 86.52$ and for benign tumors was $40.25 \pm 2.39$ and the mean HE4 between the benign group and malignant group was $P=.001$ which was significant.

Average CA125 in patients with malignant ovarian tumors was $324.98 \pm 98$ and for benign tumors was $10.22 \pm 50.11 \%$, respectively and the difference of the CA125 level between benign and malignant groups was statistically significant $(P \geq .001)$.
Table 1. Frequency Distribution of Patients With Benign Histopathology $(n=67)$

\begin{tabular}{lcc}
\hline Histopathological Type & Number & $\%$ \\
\hline Endometrioma & 20 & 29.9 \\
Follicular cyst & 8 & 11.9 \\
Teratoma & 11 & 16.4 \\
Serous cyst adenoma & 16 & 23.9 \\
Fibroma & 3 & 4.5 \\
Mucinous cyst adenoma & 7 & 10.4 \\
Hydatid cyst & 2 & 3 \\
\hline
\end{tabular}

Table 2. Frequency Distribution of Patients With Malignant Ovarian Tumor; Histopathology, Tumor Grade and Stage $(n=33)$

\begin{tabular}{lll}
\hline & Number & $\%$ \\
\hline Histopathology & & \\
Serous adenocarcinoma & & 52.6 \\
Endometrioma & 20 & 15.2 \\
Mucinous & 4 & 23.2 \\
Cyst adenocarcinoma & 8 & \\
Stage (FIGO) & & 1.6 \\
I & 2 & 30.3 \\
II & 10 & 6.57 \\
III & 19 & 0.6 \\
IV & 2 & 11.9 \\
Tumor grade & & - \\
1 & 3 & 9.90 \\
2 & - & \\
3 & 30 &
\end{tabular}

Average ROMA in patients with malignant ovarian tumors was $67.51 \pm 2.5 \%$ and for patients with benign tumors was $7.6 \pm 1.1 \%$ and the difference of the ROMA level between these 2 groups was statistically significant $(P \geq .001)$.

Table 3 shows the values of ROMA, CA125 and HE4 in patients with malignant tumors based on their different stages and grades. The difference between the average CA125 in patients with stage I-II (early stage) ovarian cancer in comparison to the levels of CA125 in patients with stage III-IV (advanced stage) ovarian cancer were not statistically significant. The average HE4 in patients with stage I-II ovarian cancer was $(P=.04)$. There was no significant difference found between the average ROMA in patients with stage I-II in comparison to patients with stage III-IV ovarian cancer $(P=.25)$.

The mean ROMA levels in patients with grades I-II ovarian cancer were not statistically significant in comparison to the ROMA levels in patients with grades III-IV ovarian cancer $(P=.59)$.

In patients with benign ovarian mass the mean CA125 in menopausal women was $23.9 \pm 14.8$ and in non-menopausal women was $55.2 \pm 12.1$. Considering $\mathrm{P}=0.058$ there were no meaningful statistical differences between 2 groups namely menopausal and non-menopausal. 
Table 3. Serum Level of ROMA, HE4 and CA125 and of Malignant Ovarian Tumor in Various Stages and Grades $(n=33)$

\begin{tabular}{lccc}
\hline & $\begin{array}{c}\text { CA125 (U/ML) } \\
\text { Mean } \pm \text { SD }\end{array}$ & $\begin{array}{c}\text { HE4 (PM) } \\
\text { Mean } \pm \text { SD }\end{array}$ & $\begin{array}{c}\text { ROMA (\%) } \\
\text { Mean } \pm \text { SD }\end{array}$ \\
\hline $\begin{array}{l}\text { Stage FIGO } \\
\text { I+II }\end{array}$ & $104.3 \pm 32.9$ & $7.38 \pm 93.110$ & $29.4 \pm 7.8$ \\
III+IV & $451.07 \pm 148$ & $130 \pm 9.373$ & $89.27 \pm 39.2$ \\
P value & .062 & .04 & .25 \\
Tumor grade & & & \\
I+II & $906.4 \pm 55$ & $94.6 \pm 55.1$ & $20.2 \pm 15.4$ \\
III & 347.107 & $296.7 \pm 94$ & $72.2 \pm 27$ \\
$P$ value & .357 & .352 & .59 \\
\hline
\end{tabular}

The mean HE4 in menopausal women was $45.3 \pm 9.7$ and in non-menopausal women $39.2 \pm 20.9$. Considering $P=.04$ there was a meaningful statistical difference between 2 menopausal and non-menopausal groups.

The mean ROMA in menopausal women was $10.8 \pm 5.6$ and in non-menopausal women was $6.97 \pm 1.38$. Considering $P=.94$ there were no meaningful statistical differences between 2 groups.

In patients with malignant ovarian mass the mean CA125 in menopausal women was $437.5 \pm 193.2$ and in non-menopausal women was $219.06 \pm 59$.9. Considering $P=.17$ the statistical difference between two groups was not meaningful.

The mean HE4 in menopausal women was $357.1 \pm 154.8$ and in non-menopausal women $204.1 \pm 84.9$. Considering $P=.2$ there was no meaningful statistical difference between two menopausal and non-menopausal groups.

The mean ROMA in menopausal women was $47.8 \pm 36$ and in non menopausal women was $86.01 \pm 48.9$. Considering $P=.16$ there were no meaningful statistical differences between two groups.

Finally, based on the ROC curve, ROMA, HE4, and CA125 values for the diagnosis of malignant ovarian tumors were compared and it was observed that they have high diagnostic value in ovarian cancer. The AUC of these 3 methods were calculated and no significant differences were observed (AUC of CA125, HE4 and ROMA were $82.4 \%, 84.1 \%, 82.4 \%$ respectively) (Figure 1 ).

In the assessment of the curves obtained, if 45.5 is considered to be the cut off value for CA125, the sensitivity would be considered as $84.8 \%$ and the specificity $74.1 \%$, respectively. With regards to $\mathrm{HE} 4$, if 50.9 is considered to be the cut off value, this test would have a sensitivity of $75.8 \%$ and specificity of $74.6 \%$. Regarding ROMA, if 9.4 is considered to be the cut off value, the sensitivity would be $75.8 \%$ and specificity would be $74.6 \%$.

In considering that several studies regarding the etiology and risk factors of non-mucinous ovarian tumors and mucinous ovarian tumors yield different results, therefore, the mean of the three tumor markers among patients with mucinous ovarian tumors were compared with non-mucinous ovarian tumors with the following result: mean CA125 in patients with non-mucinous malignant patho-

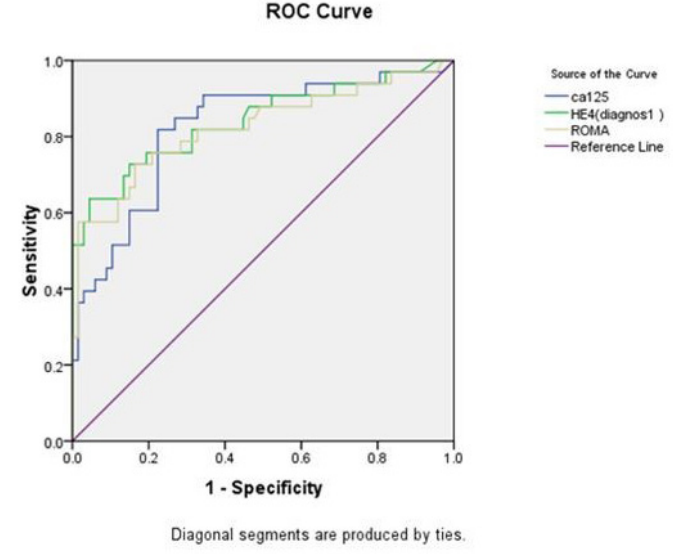

Figure 1. ROC Curve for Evaluation of 3 Tumor Makers in the Diagnosis of Ovarian Cancer.

logic ovarian malignancy was $290.5 \pm 51$ and for patients with mucinous ovarian tumor was $432.4 \pm 392$, respectively $(P=.009)$.

Mean HE4 in patients with mucinous ovarian carcinoma was $129 \pm 59$ and the mean for non-mucinous carcinoma was $111 \pm 326$ with $P=.16$ and finally the mean ROMA of mucinous ovarian carcinoma was $31.45 \pm 12$ and the mean for non-mucinous malignancies was $79 \pm 33(P=.4)$.

\section{Discussion}

HE4, as a single tumor marker, has the most sensitivity in differentiating ovarian tumors from benign masses and using both HE4 and CA125 has more exact predicting power than each of them alone (8-11). Our findings disagree with above result, while it is in accordance with findings of Van Gorp et al (10). In Van Gorp et al study ROMA, HE4 in comparison with CA125 alone, did not have much more power in diagnosing ovarian cancer. In the present study, the mean studied three tumor markers in patients with grade 1,2 ovarian cancer did not have difference with grade 3 patients. Also the mean CA125, ROMA between the 2 early stages (I,II) and advanced stage (III,IV) groups were not different but the mean HE4 marker in advanced stage was meaningfully more than early stage (I,II). Findings of the present study on grade of malignant tumors showed that despite the small number of the sample size, it did not match the findings of Van Gorp et al study (10); however in their study the level of the 3 tumor markers were significantly higher with increasing stage of the disease process.

In this present study, the 3 tumor markers ROMA, HE4 and CA125 in patients with ovarian cancer were highly significant however ROMA and HE4 are not more sensitive in differentiating malignancy before surgery in comparison to CA125. In considering the cost of these tests, it seems that it is more cost effective for patients to undergo a combined CA125 and HF4 test rather than the single CA125 test. Also, in the present study, the mean of the three tumor markers under study in patients having grade I and II ovarian cancer did not show any significant difference in comparison to patients having grade III ovarian carcinoma and also, no significant difference has been 
observed on the mean of the ROMA and CA125 between the 2 groups of patients having stage I-II ovarian cancer and patients with stage III-IV, respectively. But the mean of the tumor marker HE4 in the advanced stage of ovarian cancer was significantly higher in comparison to the early stage (I-II) of cancer.

With regards to tumor grade, results of this present study showed that the mean of these three tumor markers in patients with grade (I-II) malignant tumors, despite the small number of the sample size, yield similar results to the study conducted by Van Gorp et al (10). However, in their study the level of the 3 tumor markers mentioned above were significantly higher with increasing stage of the disease process.

\section{Conclusion}

In this study, all three tumor marker HE4, CA125 and ROMA were able to distinguish malignant from benign tumors, but the value of ROMA and HE4 in diagnosing ovarian cancer was not higher in comparison to CA125 alone. Despite the promising results in preliminary studies, in the present study, HE4 and ROMA measurements in comparison to CA125 alone was not of much help in diagnosing cancer.

\section{Ethical issues}

Ethical clearance was obtained from the Ethics Committee of Tabriz University of Medical Sciences (5/4/11853, 2014/3/15).

\section{Conflict of interests}

The authors declare no conflict of interests.

\section{Finnacial support}

Womens Reproductive Health Research Center, Tabriz University of Medical Science.

\section{Acknowledgments}

We would like to thank the authorities of Tabriz University of Medical Sciences for the scientific and ethical approval and financial support of this research. This study has been done as a thesis for specialty degree of Marzye Parizad in Women's Reproductive Health Research Center, Tabriz University of Medical Sciences (2013/4/4, No: 9117).

\section{References}

1. Abdel-Azeez HA, Labib HA, Sharaf SM, Refai AN. HE4 and mesothelin: novel biomarkers of ovarian carcinoma in patients with pelvic masses. Asian Pac J Cancer Prev. 2010;11(1):111-116.
2. Anton C, Carvalho FM, Oliveira EI, Maciel GA, Baracat EC, Carvalho JP. A comparison of CA125, HE4, risk ovarian malignancy algorithm (ROMA), and risk malignancy index (RMI) for the classification of ovarian masses. Clinics (Sao Paulo) 2012;67(5):437-441.

3. du Bois A, Rochon J, Pfisterer J, Hoskins WJ. Variations in institutional infrastructure, physician specialization and experience, and outcome in ovarian cancer: a systematic review. Gynecol Oncol. 2009;112(2):422436. doi: 10.1016/j.ygyno.2008.09.036.

4. Moore RG, Brown AK, Miller MC, et al. The use of multiple novel tumor biomarkers for the detection of ovarian carcinoma in patients with a pelvic mass. Gynecol Oncol. 2008;108(2):402-408. doi: 10.1016/j. ygyno.2007.10.017.

5. Moore RG, Jabre-Raughley M, Brown AK, et al. Comparison of a novel multiple marker assay vs the Risk of Malignancy Index for the prediction of epithelial ovarian cancer in patients with a pelvic mass. Am J Obstet Gynecol. 2010;203(3):228.e1-6. doi: 10.1016/j.ajog.2010.03.043.

6. Moore RG, McMeekin DS, Brown AK, et al. A novel multiple marker bioassay utilizing HE4 and CA125 for the prediction of ovarian cancer in patients with a pelvic mass. Gynecol Oncol. 2009;112(1):40-46. doi: 10.1016/j.ygyno.2008.08.031.

7. Nolen B, Velikokhatnaya L, Marrangoni A, et al. Serum biomarker panels for the discrimination of benign from malignant cases in patients with an adnexal mass. Gynecol Oncol. 2010;117(3):440-5. doi: 0.1016/j.ygyno.2010.02.005.

8. Petru E, Luck HJ, Stuart G, et al. Gynecologic Cancer Intergroup (GCIG) proposals for changes of the current FIGO staging system. Eur J Obstet Gynecol Reprod Biol. 2009;143(2):69-74. doi: 10.1016/j. ejogrb.2008.12.015.

9. chummerM, $\mathrm{NgWV}$, BumgarnerRE, etal.Comparative hybridization of an array of 21,500 ovarian cDNAs for the discovery of genes overexpressed in ovarian carcinomas. Gene. 1999;238(2):375-385.

10. Van Gorp T, Cadron I, Despierre E, et al. HE4 and CA125 as a diagnostic test in ovarian cancer: prospective validation of the Risk of Ovarian Malignancy Algorithm. Br J Cancer. 2011;104(5):863870. doi: 10.1038/sj.bjc.6606092.

11. Woolas RP, Xu FJ, Jacobs IJ, Yu YH, Daly L, Berchuck $A$, et al. Elevation of multiple serum markers in patients with stage I ovarian cancer. J Natl Cancer Inst. 1993;85(21):1748-1751.

Copyright $(2015$ The Author(s); This is an open-access article distributed under the terms of the Creative Commons Attribution License (http://creativecommons.org/licenses/by/4.0), which permits unrestricted use, distribution, and reproduction in any medium, provided the original work is properly cited. 